\title{
Relaxation of excited electrons in a paramagnetic electron gas: The role of spins in screening and scattering
}

\author{
I. Nagy, ${ }^{1,2}$ M. Alducin, ${ }^{3}$ J. I. Juaristi, ${ }^{4}$ and P. M. Echenique ${ }^{4,5}$ \\ ${ }^{1}$ Department of Theoretical Physics, Technical University of Budapest, H-1521 Budapest, Hungary \\ ${ }^{2}$ Donostia International Physics Center, 20080 San Sebastián, Spain \\ ${ }^{3}$ Departamento de Ingeniería Eléctrica, E.T.S.I.I., Universidad del País Vasco, Alda. Urquijo, s.n., 48013, Bilbao, Spain \\ ${ }^{4}$ Departamento de Física de Materiales, Universidad del País Vasco, Aptdo. 1072, 20080 San Sebastián, Spain \\ ${ }^{5}$ Centro Mixto CSIC-UPV/EHU, Aptdo. 1072, 20080 San Sebastián, Spain
}

(Received 26 February 2001; published 5 July 2001)

\begin{abstract}
The scattering lifetime of excited electrons close enough to the Fermi surface is investigated using the standard kinetic framework. This framework is implemented by effective, spin-dependent interactions and numerical phase-shift calculations. The nontrivial interplay between wave-mechanical interference effects in scattering and correlations in screening and dressing is discussed. Their role and relative importance are quantified via a comparative theoretical study.
\end{abstract}

DOI: 10.1103/PhysRevB.64.075101

PACS number(s): 71.10.Ca, 72.15.Lh, 71.45.Gm

\section{INTRODUCTION AND MOTIVATIONS}

Many of the laws of quantum theory have been discovered by watching the behavior of single particles being scattered, making allowed transitions and passing through different fields. Nevertheless, in condensed-matter physics, we must apply these laws to the calculation of the properties of many-body quantum systems. The model of an interacting electron gas, i.e., a system of identical fermion particles, not localized in space and in interaction with one another, represents a genuinely important problem. The understanding of the dynamics of excited electrons in this system, and thus to get a physical insight into nontrivial correlation effects, is of great theoretical and experimental interest currently. For a recent review on this field, see Ref. 1.

Consider an excited particle lying above the Fermi surface. Such a particle, the state of which is classified by its momentum distribution function, will not remain there indefinitely. It will scatter against the particles in the Fermi sphere, making real transitions and so tend to lower its energy. ${ }^{2}$ The excited state therefore has a finite scattering lifetime $(\tau){ }^{3,4}$ When the energy $(E)$ of an excited electron is close enough to the Fermi surface $\left(E_{F}\right)$ the relaxation process is mediated by electron-hole pair excitations carrying energy $(\omega)$ and momentum $(q)$. Due to the fundamental role of the Pauli exclusion principle for energetically allowed excitations, $\max (\omega)=E-E_{F}$. This is a phase-space constraint; it is essentially independent of the details of interactions between particles of a normal Fermi system. The physical relaxation process is inelastic and its characteristic time scale should depend on the details of those scatterings that give real transitions. The process is an irreversible, dissipative one in the thermodynamical sense. In other words, $\omega \operatorname{Im} \chi_{0}(q, \omega) \geqslant 0$ by causality, where the complex $\chi_{0}(q, \omega)$ is the response function of a system of independent constituents and the equality holds only for quasistatic processes. ${ }^{5}$ The factor $\operatorname{Im} \chi_{0}(q, \omega)$, the spectral function, measures the dynamical width of particle states. ${ }^{6}$

The theoretical description of scattering of two identical fermions (not necessarily electrons, i.e., nucleons) interacting through central forces is well-known, ${ }^{7,8}$ The description is based on the free-space Schrödinger equation. We may suppose-and this will be the actual case in this work-that the spin-dependent part of the two-body effective interaction is proportional to the product $\boldsymbol{\sigma}_{1} \cdot \boldsymbol{\sigma}_{2}$ of two Pauli spin operators. ${ }^{3,4,8}$ Generally, the scattering state function must be antisymmetric when all the coordinates of the two fermions (spatial and spin) are interchanged. The rate of scattering is related ${ }^{9}$ to the differential cross section $(d \sigma)$ which is defined in terms of properly determined singlet and triplet amplitudes. At these above circumstances the role of exchange appears as an interference effect in the differential cross section. $^{8}$

In the present work we shall investigate in detail the physical aspects of the effective interaction required in an implementation of the kinetic treatment of the relaxation process. ${ }^{2}$ The influence of the fermion many-body environment "around" our two-particle subsystem results in a shortrange effective interaction between them. The scattering should be described, therefore, at least via an in-medium Schrödinger equation. The effective interaction must include electrostatic screening and exchange-correlation-mediated dressing, due to the Coulomb and Pauli correlations. These may affect the scattering characteristics $(d \sigma)$ in a nontrivial manner in combination with the above-mentioned waveinterference effect.

This paper is organized as follows. In Sec. II, we present the theoretical framework for the scattering lifetime, the models for the effective interaction, and the obtained results. These latter are given analytically and numerically in the form of illustrative figures. The results, based on different approximations, are discussed in a comparative way. The last section, Sec. III, is devoted to the conclusions. We shall use atomic units $e^{2}=\hbar=m_{e}=1$ throughout this work.

\section{THEORY AND RESULTS}

The evaluation of the kinetic model for the scattering lifetime results in the following expression: ${ }^{2,3}$ 


$$
\frac{1}{\tau}=a\left(r_{s}\right)\left(E-E_{F}\right)^{2},
$$

in which the prefactor $a\left(r_{s}\right)$ depends, generally in a complicated way, on the density $n_{0}=3 /\left(4 \pi r_{s}^{3}\right)$ of the electron system. This factor is determined (for a recent implementation, see, i.e., Ref. 10) from

$$
a\left(r_{s}\right)=\frac{1}{(2 \pi)^{4}} \frac{1}{2 \pi} \int_{0}^{\pi} d \theta \int_{0}^{\pi} d \alpha \sin \alpha \frac{w(\alpha, \theta)}{\cos (\alpha / 2)},
$$

where $\alpha$ is defined via $\cos \alpha=1-\left(E_{c} / E_{F}\right), E_{c}$ is the scattering energy $E_{c} \in\left[0,2 E_{F}\right]$ in the center-of-mass (c.m.) system, and $\theta$ is the usual scattering angle. The function $w(\alpha, \theta)$ is given by the differential cross section $d \sigma\left(E_{c}, \theta\right) \equiv d \sigma$ as

$$
w(\alpha, \theta)=2 \pi\left[\frac{2 \pi}{\mu}\right]^{2} \frac{1}{2} d \sigma,
$$

in which $\mu=1 / 2$ is the reduced mass in c.m. system. The factor of $(1 / 2)$ in front of $d \sigma$ takes care of the proper normalization of the integrated cross sections for identical particles.

Supposing an effective interaction energy to our (inmedium) scattering Schrödinger equation of the form

$$
V_{e f f}(r)=V_{1}(r)-\boldsymbol{\sigma}_{1} \cdot \boldsymbol{\sigma}_{2} J(r)
$$

where $V_{1}(r)$ and $J(r)$ are regular functions and will be given below, for unpolarized scattering one can write: ${ }^{7,8}$

$$
d \sigma=\frac{1}{4} d \sigma_{s}+\frac{3}{4} d \sigma_{t r}
$$

The singlet $(s)$ and triplet $(t r)$ differential cross sections are related to the standard scattering amplitudes $f_{i}(\theta)$ as follows:

$$
\begin{gathered}
d \sigma_{s}=\left|f_{s}(\theta)+f_{s}(\pi-\theta)\right|^{2}, \\
d \sigma_{t r}=\left|f_{t r}(\theta)-f_{t r}(\pi-\theta)\right|^{2} .
\end{gathered}
$$

These amplitudes are calculated by using Eq. (4) properly, i.e., $V_{\text {eff }}(r)=V_{1}(r)+3 J(r)$ and $V_{\text {eff }}(r)=V_{1}(r)-J(r)$ for the singlet and triplet channels, respectively. ${ }^{8,11}$

The explicit implementation of the present formalism needs, in the spirit of Sec. I, physically reasonable forms for $V_{1}(r)$ and $J(r)$ to Eq. (4). More precisely, one has to consider the electrostatic screening and exchange-correlation (between the scattering pair and system particles) mediated dressing. Here we follow an inherently natural and appealing way of sorting out the role of charge-induced (Coulomb) and spin-induced (Pauli) correlations. First, we discuss the form of $J(r)$ to be used here.

According to physically motivated $^{12,13}$ and diagrammatical ${ }^{14}$ approaches, the spin-mediated part of the effective electron-electron interaction, Eq. (4), has the following form in momentum $(q)$ space:

$$
J(q)=\left[v(q) G_{a}(q)\right]^{2} \frac{\chi_{0}(q)}{1-v(q) G_{a}(q) \chi_{0}(q)} .
$$

In this equation $v(q)=4 \pi / q^{2}, G_{a}(q)$ is the spinantisymmetric static local-field correction, ${ }^{15}$ and $\chi_{0}(q)$ is the Lindhard function. ${ }^{4,6}$ The second term in the product of Eq. (8) is the so-called spin-incoherent response function. ${ }^{15} \mathrm{Mul}-$ tiplying this spin response function by $\mu_{B}^{2}$ (where $\mu_{B}$ is the Bohr magneton) one gets the Pauli spin susceptibility. ${ }^{12,16}$ It measures the sensitivity of the paramagnetic system to spinrelated perturbations and is a very important quantity, i.e., in the interpretation of experimental Knight-shift data. ${ }^{15,17}$ By tuning its denominator one can model (in an average manner) the susceptibility enhancement (around 10) observed in the so-called nearly ferromagnetic Pd metal. ${ }^{18}$

Here we adopt the parametrization given by Iwamoto and Pines for the $G_{a}(q)$ function: ${ }^{15,19}$

$$
G_{a}(q)=\frac{1}{2}\left(\frac{q^{2}}{q^{2}+\alpha_{\uparrow \uparrow}^{2}}-\frac{q^{2}}{q^{2}+\alpha_{\uparrow \downarrow}^{2}}\right),
$$

in their polarization-pseudopotential treatment. The parameters $\alpha_{\uparrow \uparrow}$ and $\alpha_{\uparrow \downarrow}$ are determined by requiring that in the limit $q \rightarrow 0$ one obtain correct compressibility and paramagnetic spin susceptibility; for further details, see Ref. 15 . We use the complete equation (9) in the first (square) term of Eq. (8) and the usual small- $q$ limit in the spin-response function to perform an inverse Fourier transformation. The obtained result is the following:

$$
J(r)=J_{0}\left[\frac{1}{\alpha_{\uparrow \uparrow}} e^{-\alpha_{\uparrow \uparrow} r}+\frac{1}{\alpha_{\uparrow \downarrow}} e^{-\alpha_{\uparrow \downarrow} r}-\frac{4}{r} \frac{e^{-\alpha_{\uparrow \uparrow} r}-e^{-\alpha_{\uparrow \downarrow} r}}{\alpha_{\uparrow \downarrow}^{2}-\alpha_{\uparrow \uparrow}^{2}}\right],
$$

where the Stoner-like prefactor has the form of

$$
J_{0}=\left(\frac{k_{F}}{2 \pi}\right)\left(1-\frac{2 k_{F}}{\pi} \frac{\alpha_{\uparrow \downarrow}^{2}-\alpha_{\uparrow \uparrow}^{2}}{\alpha_{\uparrow \uparrow}^{2} \alpha_{\uparrow \downarrow}^{2}}\right)^{-1} .
$$

Note, in passing, that the formal $\alpha_{\uparrow \downarrow} \rightarrow \infty$ corresponds to the so-called exchange-only, additional, approximation. Furhermore, $J(r)$ is a regular "potential energy" with a finite value at the origin $r=0$.

Next, in order to compare our numerical results with simple and frequently used ones ${ }^{1,20-24}$ and thus to get clear links, we outline these latter. Performing a first-order Born $\left[f(q)=(\mu / 2 \pi) V_{e f f}(q)\right.$ and $\left.q=2 \sqrt{E_{c}} \sin (\theta / 2)\right]$ calculation with $V_{1}(r)=(1 / r) \exp (-\beta r)$ and $J(r)=0$ in a classical treatment, i.e., neglecting the interference terms in Eqs. (6) and (7), one can obtain

$$
a\left(r_{s}\right)=\frac{1}{\pi v_{F}} \frac{1}{\beta^{3}}\left(\arctan \gamma+\frac{\gamma}{\gamma^{2}+1}\right),
$$

as was shown earlier, ${ }^{20,21} \gamma=\left(2 v_{F}\right) / \beta$. Using the same approximation but with perturbative interference terms (exchange in scattering), the result becomes ${ }^{22-24}$ 


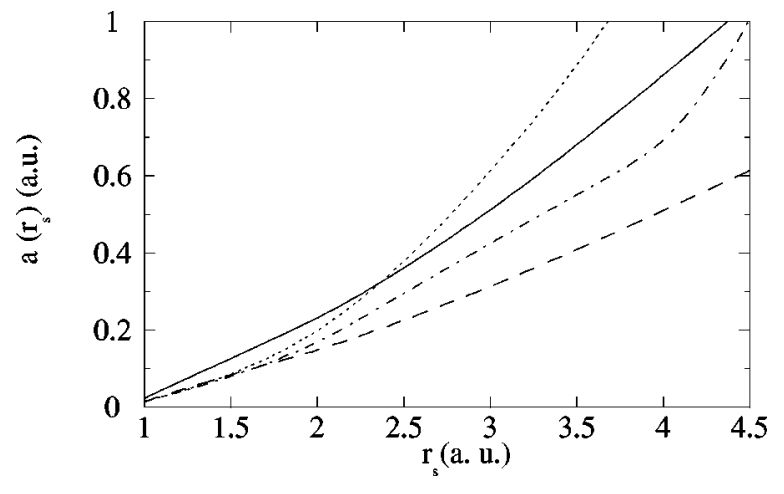

FIG. 1. Numerically determined $a\left(r_{s}\right)$ functions to Eq. (1). All the curves are based on the same linear Thomas-Fermi term $V_{1}(r)$ in the effective interaction; see Eq. (4). The solid and dashed curves are obtained with $J(r)=0$ in Eq. (4) and refer to classical (no interference in scattering) and exchange-mediated (interference in scattering) approximations. The dotted and dash-dotted curves are obtained with $J(r) \neq 0$ in Eq. (4), and include the corresponding interference terms. The dotted curve refers to the exchange-only approximation; see Eq. (11).

$$
\begin{aligned}
a\left(r_{s}\right)= & \frac{1}{\pi v_{F}} \frac{1}{\beta^{3}}\left[\arctan \gamma+\frac{\gamma}{\gamma^{2}+1}\right. \\
& \left.-\frac{1}{\sqrt{\gamma^{2}+2}} \arctan \left(\gamma \sqrt{\gamma^{2}+2}\right)\right] .
\end{aligned}
$$

In standard evaluations of Eqs. (12) and (13), the usual Thomas-Fermi screening parameter $\beta^{2}=\left(4 v_{F}\right) / \pi$ is employed. Both expressions show their special sensitivity ${ }^{10}$ to the precise form of screening $(\beta)$. Note that in a recent theoretical interpretation of nonequilibrium electron dynamics ${ }^{25}$ in noble metals a reduced $\left(\beta_{r}\right)$ static screening was predicted with $\beta_{r}=0.73 \beta$. At metallic densities $\left(r_{s} \simeq 2\right)$, Eq. (13) gives, roughly, about a $40 \%$ decrease in $a\left(r_{s}\right)$ with respect to Eq. (12), if we use the Thomas-Fermi value for $\beta$. The interference term may be, therefore, an important ingredient in quantitative predictions for the scattering lifetime $\tau$ in an electron gas.

We have performed numerical calculations with the a priori given Thomas-Fermi potential energy $V_{\text {eff }}(r)=V_{1}(r)$ using standard partial-wave expansions for the scattering amplitudes:

$$
f(\theta)=\frac{1}{\sqrt{E_{c}}} \sum_{l=0}^{\infty}(2 l+1) e^{i \delta_{l}} \sin \delta_{l} P_{l}(\cos \theta),
$$

in which $\delta_{l}\left(E_{c}\right)$ are the usual phase shifts. The solid and dashed curves in Fig. 1 are the so-called phase-shift-based equivalents of Eqs. (12) and (13), respectively. Both curves give, in comparison with the above perturbative predictions, reductions of about $50 \%$ at metallic densities. Therefore, the level of scattering treatments is an important ingredient, too.

The role of the wave-mechanical interference term in fermion scattering is, similarly to the above-discussed perturbative estimation, quite notable. The other two curves of Fig. 1 refer to numerical phase-shift calculations (with the interfer- ence terms) and are obtained by using different approximations to the $J(r)$ function in Eq. (4). The dotted curve is based on the exchange-only while the dash-dotted one on the complete form of $J(r)$; see Eqs. (10) and (11). The nontrivial role of correlation effects in scatterings and screenings is clearly visible. The exchange-only approximation, with the Thomas-Fermi form for $V_{1}(r)$, lifts the dotted curve close to (or above) the values of the solid one at metallic densities. Therefore, the linear-response-based observation of Kleinman $^{26}$ on the twofold (compensating) role of exchanges is verified in this case, although with systematically reduced values for the inverse scattering lifetime [cf. Eqs. (12) and (13)].

We have discussed, in this section, analytical and numerical results, presupposing a simple Thomas-Fermi form for $V_{1}(r)$. This corresponds to a quasiclassical, linear response approximation. Recently, we have developed a parametric nonlinear treatment to describe the screening of a light $m$ $=m_{e}$ negative charge $(Z=-1)$ in an electron gas. ${ }^{10}$ This Hartree-type treatment gave the following form for $V_{1}(r)$ :

$$
\begin{aligned}
V^{1}(r)= & -\frac{Z}{r} \frac{1}{4 \alpha_{1} \alpha_{2}} e^{-\alpha_{1} r}\left[\left(\alpha_{1}+\alpha_{2}\right)^{2} e^{\alpha_{2} r}\right. \\
& \left.-\left(\alpha_{1}-\alpha_{2}\right)^{2} e^{-\alpha_{2} r}\right]
\end{aligned}
$$

in which the factors $\alpha_{1}$ and $\alpha_{2}$ are given by $\left(\omega_{p}^{2}=4 \pi n_{0}\right)$

$$
\begin{aligned}
& \alpha_{1}=\left[\frac{b}{2 \lambda}+\frac{\omega_{p}}{\sqrt{\lambda}}\right]^{1 / 2}, \\
& \alpha_{2}=\left[\frac{b}{2 \lambda}-\frac{\omega_{p}}{\sqrt{\lambda}}\right]^{1 / 2} .
\end{aligned}
$$

The parameters $b$ and $\lambda$ were fixed and determined, respectively, via the average dynamics of electrons at the Fermi level $\left[b=(4 / 3) v_{F}^{2}\right]$ and the electronic cusp condition $(\mu$ $=1 / 2)$ for the induced hole density at zero $(r=0)$ separation $\left[\lambda=\lambda\left(r_{s}\right)\right]$. The potential, described in such a way, was compared $^{10}$ with the Thomas-Fermi form and an essential reduction of screening at short distances was established (cf. Ref. 25). Furthermore, an electrostatic energy, defined as $\left[V_{1}(r)-(1 / r)\right](r=0)$ with $Z=-1$, has a very reasonable, Wigner-like, character at low densities in our nonlinear treatment. In fact, it is easy to show that it scales as $-r_{s}^{-1}$. Without the cusp constraint (namely, with constant $\lambda$ ) one would get the usual $-r_{s}^{-3 / 4}$-like asymptotic behavior of the random-phase approximation. ${ }^{27}$ Note that at low densities $G_{a}(q) \rightarrow 0$ [see Eqs. (8) and (9)] according to Ref. 15. Physically, the Coulomb correlations are expected to dominate Pauli correlations. The Wigner limit is governed by a statistics-independent, classical electrostatics. ${ }^{3}$

On the other hand, as our kinetic model for $1 / \tau$ is based on the concept of an embedded "two-particle-subsystem," any construction for screened interactions using the "probeparticle plus environment" picture is not obvious a priori. Consider the interaction energy [defined as $E(r)$ ] between two point charges of a dimer $\left(Z_{1}=Z_{2}\right)$ immersed statically in an electron gas. In the perturbative, linear-response ap- 


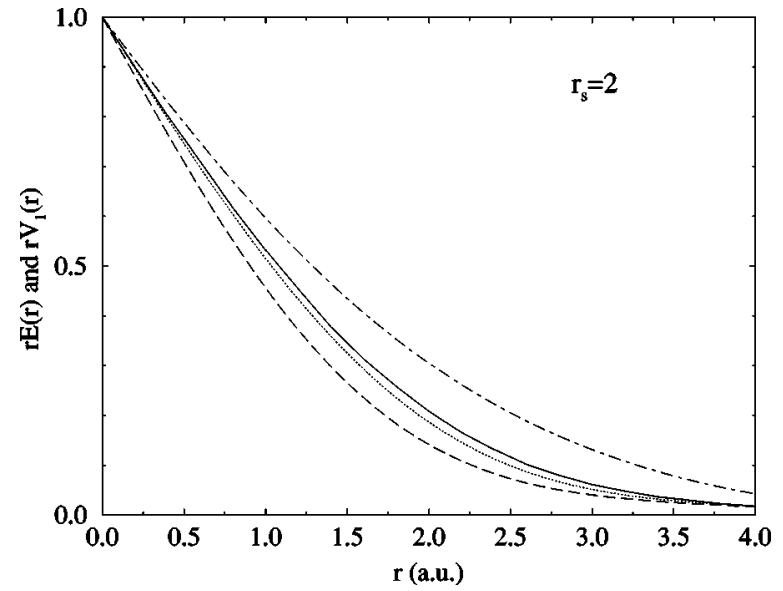

FIG. 2. The interaction energy [denoted as $r E(r)$ and plotted by a solid curve] between static antiprotons of an embedded dimer in an electron gas of $r_{s}=2$. The dashed and dotted curves refer to potential energies [plotted as $\left.r V_{1}(r)\right]$ of a unit and a one-half negative charge in the screened field of an antiproton and a negative alpha $\left(Z_{1}=-2\right)$ particle, respectively. The dash-dotted curve refers to Eq. (15), for the nonlinear electron-electron interaction.

proximation $E(r)$ is equal (at a given intercharge distance $r$ in the dimer) with the electrostatic energy of one charge sitting in the electrostatic (linearly screened) potential of the second charge. ${ }^{28}$ Clearly, the nonlinearity problem in $E(r)$ needs investigation.

Two close repulsive particles will strongly repel the surrounding electrons, and they will tend to polarize the medium quite symmetrically. ${ }^{29}$ In order to get a quantitative insight into this problem, and thus a further justification of Eq. (15), we apply an auxiliary example: namely, a dimer consisting of two antiprotons $\left(Z_{1}=Z_{2}=-1\right)$. Due to an efficient numerical method, established recently for dimers by using $^{30}$ density functional theory, we can calculate the $E(r)$ $=(1 / r)+E_{e m}(\bar{p} \bar{p})-2 E_{e m}(\bar{p})$ quantity. Here, $E_{e m}(\bar{p} \bar{p})$ and $E_{e m}(\bar{p})$ are the embedding $(e m)$ energies for a dimer $(\bar{p} \bar{p})$ and a single antiproton $(\bar{p})$. The result obtained for $r E(r)$ by the nonlinear, self-consistent calculation (performed at the Hartree level, for $r_{s}=2$ ) is exhibited in Fig. 2, by a solid curve. The other curves are based on nonlinear (Hartree) screening calculations for an antiproton and a negative alpha $\left(Z_{1}=-2\right)$ particle. In both cases, $\mu=1$, of course. The curves (dashed and dotted) refer to the potential energies of a negative unit and a negative one-half probe charge in the obtained screened fields, respectively.

The comparison shows that the elementary linearresponse prediction (see above, Ref. 28) is applicable with an acceptable rigor even to nonlinearly screened interactions, at least in the case of repulsive embedded charges. This gives further support to use Eq. (15) in electron-electron interactions. The nonlinear result [obtained via Eq. (15)] for our $r V_{1}(r)$ is plotted in Fig. 2, by a dash-dotted curve. The reduced-mass effect and the average dynamics result in a notable change, in comparison with the static-charge screening. The usual, linear-response-based Thomas-Fermi approximation for $r V_{1}(r)$ would be below all the curves of

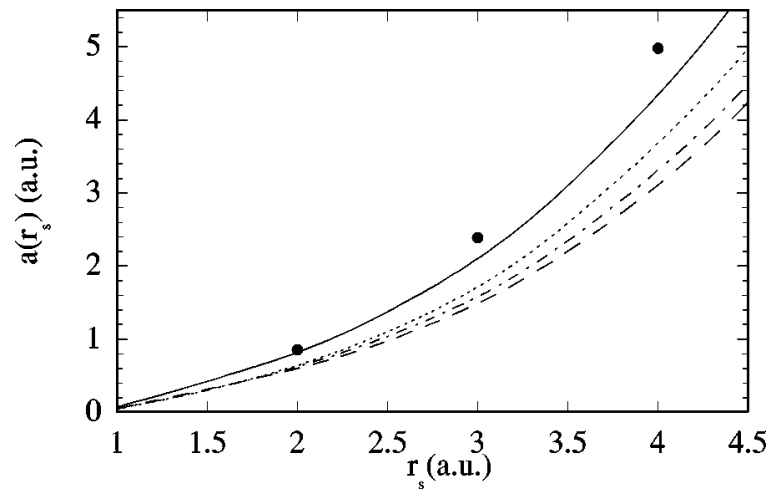

FIG. 3. The same as in Fig. 1, but $V_{1}(r)$ from Eq. (15) is used in every case, systematically. The black dots refer to numerical results obtained via an enhanced spin susceptibility in the spindependent part $J(r)$.

Fig. 2. A more rigorous treatment for this effective interaction would require a Bethe-Salpeter-type , $^{4,31}$ consideration, in a restricted phase space around $v_{F}$, and is out of the scope of the present paper.

After these clarifications of the nontrivial role of the scattering descriptions, the physically reasonable forms of nonlinear effective screenings, the special influence of average dynamics, and spin-mediated dressing, we present our consistent results. The calculations for the scattering lifetime, Eq. (1), have been performed by using Eq. (4) with Eqs. (10) and (15) together with Eqs. (6) and (7). Figure 3, similarly to Fig. 1, contains four curves. The solid and dashed curves are based on Eq. (15) with $J(r)=0$ in Eq. (4), without and with the wave-mechanical interference terms, respectively. This interference effect turns out to be important, but with reduced effectiveness (see Fig. 1). This is due to the stronger repulsive nature of Eq. (15) in comparison with a ThomasFermi estimation for $V_{1}(r)$.

The other two curves are based on Eq. (4) with the inclusion of $J(r)$. The dotted curve refers to the so-called exchange-only, additional, approximation, while the dashdotted curve to the complete form of $J(r)$. The differences between these last results are small; compare them with the corresponding ones in Fig. 1. The intricate interplay between proper scattering and screening and dressing is established. Note, however, that all the curves of Fig. 3 give essential reductions in lifetimes, in comparison with those of Fig. 1.

Finally, as we already mentioned after Eq. (8), by tuning the spin susceptibility one can model (in an average manner) the influence of its enhancements. Illustrative results of this tuning are exhibited in Fig. 3 at $r_{s}=2, r_{s}=3$, and $r_{s}=4$. The black dots refer to simple multiplication of Eq. (11) (and in such a way that they become the enhanced equivalents of the dash-dotted curve) by a factor of 10 . These results of Fig. 3 show that, although the precise form of the electrostatic screening is a strongly determining factor ${ }^{10,25}$ in scatteringlifetime calculations for a paramagnetic electron gas, a quantitative prediction must rest on the details. In this respect, a scattering-lifetime measurement for the nearly ferromagnetic ${ }^{18,32}$ Pd metal could help to substract informa- 
tion on the possible role of susceptibility-enhancement effects in the relaxation process.

The curves in Fig. 3 show a noticable reduction of the lifetime (around a factor of 2) compared with the commonly used perturbative result, Eq. (12). Recent experimental results, obtained for the low-energy range $E-E_{F}<1 \mathrm{eV}$, seem to show this kind of tendency. ${ }^{33,34}$ These experimental predictions were based on time-resolved two-photon photoemission measurement ${ }^{33}$ for an $\mathrm{Al}$ target and on a spaceresolving technique ${ }^{34}$ for noble metals $(\mathrm{Cu}, \mathrm{Ag})$, respectively (see also Ref. 25). Notable reductions in the lifetime, in comparison with the perturbative result of Eq. (12), have been deduced, using Eq. (1) in order to interpret the measured data. On the other hand, for the noble metals the influence of $d$-band electrons can be important, as was shown (using a self-energy method) for higher energies. ${ }^{1}$ Additional lifetime experiments, for the important low-energy (Fermi liquid) range and free-electron-like metals ( $\mathrm{Al}, \mathrm{Mg}$, and alkalis), would help to understand those theoretical details that are related to the dynamics of correlated fermions.

\section{CONCLUSIONS}

We have investigated the details of scattering and screening and dressing approximations required in a practical implementation of the standard theoretical framework for the scattering lifetime of excited electrons. The relative importance of these ingredients and their interplay are clarified. The twofold role of spins in effective interactions and wavemechanical interference terms is quantified. The theoretical results are discussed in a comparative way. Comparing with commonly applied perturbative results for the electron gas, notable reductions in the lifetime were found in a nonlinear construction for screening. Agreement with experimental lifetime predictions from different sources are established. The possible role of the spin-susceptibility enhancement is pointed out.

The developed practical implementation may have a further application, in combination with the density-of-state effects, in scattering-lifetime calculations for ferromagnetic metals. ${ }^{35}$ It was pointed out that despite the qualitative success of the single-particle picture, one ought to consider many-body effects carefully ${ }^{36,37}$ for these important targets. Furthermore, recent theoretical studies on spin-polarized electron energy loss spectroscopy signal the importance of spin-related inelastic exchange effects to the complete description of the relaxation process. ${ }^{38,39}$ The spin-dependent electron scattering dynamics is the basic theoretical ingredient to understand information obtained by electron-pair emission techniques for ferromagnets. ${ }^{40}$

On the other hand, in an initially spin-polarized electron gas (e.g., a ferromagnetic metal) the response to an electromagnetic perturbation consists of coupled charge and spin fluctuations. The theoretical attempts, based on extensions of the concept of Kukkonen and Overhauser ${ }^{12}$ to infinitesimally ${ }^{41}$ or strongly polarized ${ }^{42-44}$ systems, revealed the importance of a unified treatment for the coupled problem. Clearly, the susceptibilities should depend, through spin-dependent local-field factors, on the degree of initial polarizations. This fact may provide an interesting problem to the physical process examined.

The many-body problem is a topic in its own right, with its own characteristic methods. Here we used physical approximations to treat the actual physical phenomenon, the scattering lifetime of excited electrons in a paramagnetic electron gas. As we already mentioned, a more formal fieldtheoretical attempt would require the application of the twoparticle Green function, i.e., a careful investigation of the Bethe-Salpeter equation in a fermion medium with inherent charge and spin degrees of freedom.

\section{ACKNOWLEDGMENTS}

The work of one of us (I.N.) has been supported by the OTKA (Grant Nos. T029813 and T034363). M.A., J.I.J., and P.M.E. acknowledge support from UPV/EHU and Eusko Jaurlaritza, Ministerio de Educación y Cultura (Grant No. PB97-0636) and Iberdrola S.A. We are indebted for very useful discussions to R. Díez Muiño.
${ }^{1}$ P.M. Echenique, J.M. Pitarke, E.V. Chulkov, and A. Rubio, Chem. Phys. 251, 1 (2000).

${ }^{2}$ D. Pines and P. Nozières, The Theory of Quantum Liquids (Benjamin, New York, 1966).

${ }^{3}$ G. D. Mahan, Many-Particle Physics (Plenum Press, New York, 1981).

${ }^{4}$ A. L. Fetter and J. D. Walecka, Quantum Theory of ManyParticle Systems (McGraw-Hill, New York, 1971).

${ }^{5}$ L.P. Kadanoff and P.C. Martin, Ann. Phys. (N.Y.) 24, 419 (1963).

${ }^{6}$ J. Lindhard, K. Dan. Vidensk. Selsk. Mat. Fys. Medd. 28, 8 (1954).

${ }^{7}$ B. H. Brandsen and C. J. Joachain, Quantum Mechanics (Pearson Education Ltd, Harlow, 1989).

${ }^{8}$ G. R. Satcher, Direct Nuclear Reactions (Clarendon Press, Oxford, 1983).
${ }^{9}$ R. Shankar, Principles of Quantum Mechanics (Plenum Press, New York, 1980).

${ }^{10}$ I. Nagy, J.I. Juaristi, and P.M. Echenique, Phys. Rev. B 63, 035102 (2000), and references therein.

${ }^{11}$ J. D. Walecka, Theoretical Nuclear and Subnuclear Physics (Oxford University Press, New York, 1995).

${ }^{12}$ C.A. Kukkonen and A.W. Overhauser, Phys. Rev. B 20, 550 (1979); D.R. Penn, ibid. 22, 2677 (1980).

${ }^{13}$ Y. Takada, Phys. Rev. B 47, 5202 (1993); C.F. Richardson and N.W. Ashcroft, ibid. 55, 15130 (1997).

${ }^{14}$ G. Vignale and K.S. Singwi, Phys. Rev. B 32, 2156 (1985).

${ }^{15}$ N. Iwamoto and D. Pines, Phys. Rev. B 29, 3924 (1984).

${ }^{16}$ D.R. Hamann and A.W. Overhauser, Phys. Rev. 143, 183 (1966).

${ }^{17}$ I. Nagy, B. Apagyi, J.I. Juaristi, and P.M. Echenique, Phys. Rev. B 60, R12 546 (1999). 
${ }^{18}$ W. Jones and N. H. March, Theoretical Solid State Physics (Dower, New York, 1985).

${ }^{19}$ T.K. Ng and K.S. Singwi, Phys. Rev. B 34, 7743 (1986).

${ }^{20}$ J.J. Quinn and R.A. Ferrell, Phys. Rev. 112, 812 (1958).

${ }^{21}$ R.H. Ritchie, Phys. Rev. 114, 644 (1959).

${ }^{22}$ R.H. Ritchie and J.C. Ashley, J. Phys. Chem. Solids 26, 1689 (1965).

${ }^{23}$ C.A. Kukkonen and H. Smith, Phys. Rev. B 8, 4601 (1973).

${ }^{24}$ A.H. MacDonald and D.J.W. Geldart, Can. J. Phys. 60, 1016 (1982).

${ }^{25}$ N. Del Fatti et al., Phys. Rev. B 61, 16956 (2000).

${ }^{26}$ L. Kleinmann, Phys. Rev. B 3, 2982 (1971).

${ }^{27}$ Y. Wang and J.P. Perdew, Phys. Rev. B 44, 13298 (1991).

${ }^{28} \mathrm{~N}$. H. March, in Theory of the Inhomogeneous Electron Gas, edited by S. Lundquist and N. H. March (Plenum Press, New York, 1983).

${ }^{29}$ D.N. Lowy and G.E. Brown, Phys. Rev. B 12, 2138 (1975).

${ }^{30}$ R. Díez Muiño and A. Salin, Phys. Rev. B 60, 2074 (1999); 62, 5207 (2000); R. Díez Muiño (private communication).

${ }^{31}$ A. M. Zagoskin, Quantum Theory of Many-Body Systems
(Springer-Verlag, New York, 1998).

${ }^{32}$ P. Fazekas, Lecture Notes on Electron Correlation and Magnetism (World Scientific, Singapore, 1999).

${ }^{33}$ M. Bauer, S. Pawlik, and M. Aeschliman, Proc. SPIE 3272, 201 (1998).

${ }^{34}$ L. Bürgi, O. Jeandupeux, H. Brune, and K. Kern, Phys. Rev. Lett. 82, 4516 (1999).

${ }^{35}$ E. Zarate, S.P. Apell, and P.M. Echenique, Phys. Rev. B 60, 2326 (1999).

${ }^{36}$ F. Aryasetiawan and K. Karlsson, Phys. Rev. B 60, 7419 (1999).

${ }^{37}$ K.N. Altmann et al., Phys. Rev. B 61, 15661 (2000).

${ }^{38}$ J. Hong and D.J. Mills, Phys. Rev. B 59, 13840 (1999).

${ }^{39}$ R. Saniz and S.P. Apell, Phys. Rev. B 63, 014409 (2000).

${ }^{40}$ S.N. Samarin, J. Berakdar, O. Artamonov, and J. Kirschner, Phys. Rev. Lett. 85, 1746 (2000).

${ }^{41}$ S. Yarlagadda and G.F. Giuliani, Phys. Rev. B 49, 7887 (1994); 61, 12556 (2000).

${ }^{42}$ K.S. Yi and J.I. Quinn, Phys. Rev. B 54, 13398 (1996).

${ }^{43}$ D.C. Marinescu and J.I. Quinn, Phys. Rev. B 56, 1114 (1997).

${ }^{44}$ M. Polini and M.P. Tosi, Phys. Rev. B 63, 045118 (2001). 